\title{
A REVIEW OF SALMONELLA SURVEILLANCE IN NEW SOUTH WALES, 1998-2000
}

\section{Tony Merritt and Leanne Unicomb Hunter Public Health Unit}

Salmonella is the most common bacterial cause of gastrointestinal disease notifiable in New South Wales. ${ }^{1}$ The objectives for Salmonella surveillance as listed in the NSW Notifiable Diseases Manual are: to identify the source of infection and to prevent further cases; and to monitor the epidemiology to inform the development of better prevention strategies. ${ }^{2}$ This article reviews the process of Salmonella surveillance in NSW. The system is described and an evaluation of selected attributes including system simplicity, data completeness and timeliness of notification is presented.

This study was conducted as part of a review of foodborne disease surveillance in NSW for the period 1998-2000 by members of OzFoodNet based at the Hunter Public Health Unit. OzFoodNet is a national network established by the Commonwealth Department of Health and Ageing in 2000 to enhance foodborne disease surveillance in Australia.

\section{METHODS}

\section{Evaluation}

The guidelines for the evaluation of surveillance systems developed by the Centers for Disease Control and Prevention, Atlanta were used. ${ }^{3}$ The attributes of surveillance systems-simplicity, data completeness and timeliness of notification-were evaluated. These attributes were selected because of their importance to Salmonella surveillance.

Salmonella notifications with a date of onset of illness between 1 January 1998 and 31 December 2000 were extracted from the Notifiable Diseases Database (NDD) using the Health Outcomes Information Statistical Toolkit (HOIST), a data access and analysis facility maintained by the Centre for Epidemiology and Research, NSW Department of Health. A total of 4,642 notifications were obtained for this period.

Completeness of the data was assessed by examining field completion for each of the mandatory data items, except for name, as this information was not available through HOIST. Completeness of the recording of the results of sub-typing (serovar or phage type) was also assessed for each notification. Mandatory fields are those that must be completed for every notification that is recorded in NDD. ${ }^{2}$ The completeness of the data for 2000 was further assessed by comparing NDD data with the database maintained by the National Enteric Pathogens Surveillance Scheme.

As there is no field in NDD to record the date that Salmonella serovar and phage type results are received by the public health unit, the timeliness of this information was assessed by a manual examination of paper records held at the Hunter Public Health Unit for all Salmonella notifications for the six months July-December 2001, based on specimen collection date. This period was chosen to allow comparison with the electronic transmission of data, which had not been available prior to 2001. There were 59 notifications received in this period.

These data were analysed in Microsoft Excel.

\section{RESULTS}

\section{Description of current notification system}

The current notification system is a passive surveillance system, with laboratories obliged by the NSW Public Health Act 1991 to notify all laboratory-confirmed cases of Salmonella. Notifications are made directly to public health units (PHUs), which are responsible for both the initial collection of data and for instigating an appropriate response. Laboratories notify their local PHU about cases. If the case is resident outside the PHU's area health service the notification is passed to the relevant PHU. Information is usually mailed to the PHU by the laboratory, as there is no capacity for electronic transfer of this information at present. At the PHU, the standard set of data required by NDD is manually entered. These include: demographic information for the person, pathogen details, date for onset of illness, date of notification by the laboratory, and date of notification receipt at the PHU. For cases that are part of an outbreak, there is limited capacity to link them on the database and no opportunity to record information on suspected food vehicles, contributing factors, or other summary data.

Data in NDD is transferred electronically from the PHUs to the NSW Department of Health on a daily basis, and from there data that has been de-identified is sent electronically to the Communicable Diseases Network Australia to contribute to the National Notifiable Disease Surveillance System (NNDSS). Selected data from this system is then sent to the World Health Organization.

\section{Serovar testing and phage typing}

Identification of Salmonella isolates to the level of serovar and phage type involves a complex network of laboratories. Most isolates are sent to the Institute of Clinical Pathology and Medical Research (IPCMR) in Sydney for serovar testing, but some samples are sent directly to Queensland Health Scientific Services in Brisbane, the Institute of Medical and Veterinary Science (IMVS) in Adelaide, or the Microbiological Diagnostic Unit (MDU) in Melbourne. Most phage typing is done at the MDU, but the IMVS also types some isolates.

Most serovar and phage type results are collated at ICPMR. Hard copies of these results are forwarded to the Western Sydney Public Health Unit and then mailed from there to local PHUs for action and entry on to the NDD. ICPMR also enters these results on to an electronic spreadsheet. 
From 2001, extracts from the spreadsheet have been emailed to the Hunter Public Health Unit by ICPMR, and from 2002 relevant extracts have been emailed to all PHUs by the Communicable Diseases Branch of the NSW Department of Health.

PHUs can only access their own data on NDD, which includes personal identification details. De-identified NDD data is available through HOIST for all of NSW but access requires some proficiency with the statistical software SAS.

Summary data is published in the NSW Public Health Bulletin and is also available from the National Enteric Pathogens Surveillance Scheme (NEPSS) in quarterly and annual hard copy reports. Electronic updates of aggregated data are also distributed by the NEPSS to OzFoodNet every month.

\section{Evaluation}

Simplicity

The NDD is relatively simple to use. It requires limited training, has good quality assurance features at the data entry stage, and the case definitions are clear. However, the overall notification system is complex with multiple data sources and decentralised and duplicated data entry.

\section{Data Completeness}

Table 1 presents the number and percentage of missing or invalid entries for each of the mandatory data fields in NDD except for name. There was little missing or invalid data for most items. The exception was the field describing whether the person was Aboriginal, which was incomplete for 35 per cent of cases.

Completeness of serovar and phage type results was also assessed. Approximately eight percent of Salmonella isolates had no serovar recorded on NDD (Table 2). Completeness of phage type details was poor in 1998 and had improved substantially by 2000 (Table 3).

The NEPSS database records 1,455 cases of Salmonella in NSW for 2000, 62 (4.5 per cent) more than recorded in the NDD for the same period. The NEPSS data was also more complete with phage types recorded for 100 per cent of $S$. Enteritidis, $S$. Hadar and $S$. Virchow isolates and 98 per cent of $S$. Typhimurium isolates from NSW. ${ }^{4}$

\section{Timeliness}

The timeliness of Salmonella serovar and phage type information is presented in Table 4. Receipt of hard copy serovar and phage type data at the Hunter PHU was not timely with lengthy delays between the availability of

\section{TABLE 1}

NUMBER OF MISSING AND INVALID ENTRIES FOR MANDATORY DATA FIELDS IN THE NOTIFIABLE DISEASES DATABASE, NSW, 1998-2000 N=4642

\begin{tabular}{|lrr|}
\hline Field & $\begin{array}{c}\text { Missing or invalid entry } \\
\text { N }\end{array}$ & $\begin{array}{c}\text { \% } \\
\text { Age }\end{array}$ \\
Sex & 8 & 0.2 \\
Aboriginality & 32 & 0.7 \\
Postcode & 2983 & 64.3 \\
Received date & 6 & 0.1 \\
Disease name & 0 & 0 \\
\end{tabular}

\section{TABLE 2}

COMPLETENESS OF SALMONELLA SEROVAR RECORDS, NOTIFIABLE DISEASES DATABASE, NSW, 1998-2000

\begin{tabular}{|lcc|}
\hline Year & \multicolumn{1}{c}{$\begin{array}{c}\text { Salmonella } \\
\text { notifications } \\
\boldsymbol{N}\end{array}$} & $\begin{array}{c}\text { Serovar } \\
\text { recorded } \\
\%\end{array}$ \\
\hline 1998 & 1811 & 93.0 \\
1999 & 1438 & 90.3 \\
2000 & 1393 & 92.5 \\
Total & 4642 & 92.0 \\
\hline Source: & Communicable Diseases Branch, Notifiable \\
& Diseases Database (HOIST), Centre for \\
& Epidemiology and Research, NSW Department of \\
& Health. & \\
\hline
\end{tabular}

\section{TABLE 3}

COMPLETENESS OF SALMONELLA PHAGETYPE RECORDS, NOTIFIABLE DISEASES DATABASE, NSW, 1998-2000

\begin{tabular}{|c|c|c|c|c|c|c|}
\hline \multirow[b]{2}{*}{$\begin{array}{l}\text { Salmonella } \\
\text { serovar }\end{array}$} & \multicolumn{2}{|c|}{1998} & \multicolumn{2}{|c|}{1999} & \multicolumn{2}{|c|}{2000} \\
\hline & $\begin{array}{c}\text { Notifications } \\
\text { N }\end{array}$ & $\begin{array}{c}\text { Phage type } \\
\text { recorded } \\
\%\end{array}$ & $\begin{array}{c}\text { Notifications } \\
\text { N }\end{array}$ & $\begin{array}{c}\text { Phage type } \\
\text { recorded } \\
\%\end{array}$ & $\begin{array}{c}\text { Notifications } \\
\qquad \mathbf{N}\end{array}$ & $\begin{array}{c}\text { Phage type } \\
\text { recorded } \\
\%\end{array}$ \\
\hline S. Bovismorbificans & 41 & 19.5 & 22 & 13.6 & 39 & 51.3 \\
\hline S. Enteritidis & 92 & 53.2 & 88 & 57.9 & 55 & 78.2 \\
\hline S. Hadar & 14 & 0 & 4 & 0 & 18 & 50 \\
\hline S. Heidelberg & 8 & 0 & 3 & 0 & 13 & 30.8 \\
\hline S. Typhimurium & 852 & 73.6 & 663 & 79.6 & 691 & 89.4 \\
\hline S. Virchow & 119 & 0 & 53 & 0 & 54 & 42.6 \\
\hline
\end{tabular}

Source: Communicable Diseases Branch, Notifiable Diseases Database (HOIST), Centre for Epidemiology and Research, NSW Department of Health. 
TABLE 4

SALMONELLA NOTIFICATION MILESTONES FROM THE DATE OF SPECIMEN COLLECTION, HUNTER PUBLIC HEALTH UNIT, NSW, JULY-DECEMBER 2001

\begin{tabular}{|c|c|c|c|}
\hline \multirow[t]{2}{*}{ Milestone } & \multicolumn{2}{|c|}{ Elapsed days } & \multirow{2}{*}{$\begin{array}{l}\text { Number of notifications included } \\
\text { (required dates available) }\end{array}$} \\
\hline & Median & Range & \\
\hline \multicolumn{4}{|l|}{ Hard copy data } \\
\hline Initial species report printed at laboratory & 5 & $2-18$ & 48 \\
\hline Initial species report received at HPHU & 7 & $3-20$ & 50 \\
\hline Serovar report printed at laboratory (ICPMR) & 9 & $6-57$ & 47 \\
\hline Serovar report received at HPHU & 22 & $14-72$ & 47 \\
\hline Phage type report printed at laboratory (MDU, IMVS) & 23.5 & $14-80$ & 31 \\
\hline Phage type report received HPHU & 42 & 3-97 & 31 \\
\hline \multicolumn{4}{|l|}{ Electronic data ${ }^{\#}$} \\
\hline Serovar entered on ICPMR database & 10 & $7-14$ & 15 \\
\hline Phage type entered on ICPMR database & 26.5 & $7-62$ & 30 \\
\hline \multicolumn{4}{|c|}{$\begin{array}{l}\text { \# Results from samples processed at ICPMR are entered on an electronic spreadsheet and extracts are then emailed to the } \\
\text { Hunter PHU. Delay from entry on ICPMR database to receipt at the HPHU is up to two days }\end{array}$} \\
\hline \multicolumn{4}{|c|}{ HPHU = Hunter Public Health Unit } \\
\hline \multicolumn{4}{|c|}{ ICPMR = Institute of Clinical Pathology and Medical Research } \\
\hline \multicolumn{4}{|c|}{ IMVS = Institute of Medical and Veterinary Science } \\
\hline \multicolumn{4}{|l|}{ MDU = Microbiological Diagnostic Unit. } \\
\hline \multicolumn{4}{|l|}{ Source: Hunter Public Health Unit. } \\
\hline
\end{tabular}

serovar and phage type reports at the laboratory and their receipt at the PHU.

To assess the potential impact of electronic transmission of data, the time between the date of specimen collection and the date the isolate results were entered on the electronic spreadsheet at ICPMR was calculated (Table 4). The date of entry was used to assess timeliness as the date the electronic spreadsheet was received at the Hunter Public Health Unit was not recorded. In practice the maximum time between data entry and receipt was two days. Dates were available for 30 of the 37 isolates analysed to phage type level and 15 of the 17 isolates analysed to serovar level. Five isolates were excluded, as they were unable to be typed. Electronic transmission of data resulted in serovar and phage type data being available at the Hunter Public Health Unit approximately two weeks earlier than was the case with hard copy results.

\section{DISCUSSION}

The notification system for Salmonella in NSW is complex. The system is not a useful repository for outbreak information and consequently dedicated databases for outbreak data have been developed. Databases that record serovar and phage type details are separately maintained at the state and national levels and these are not integrated with the NDD.

The completeness of the data for the mandatory data fields in NDD was good with the exception of the field describing Aboriginality. There was also incomplete recording of serovar and phage type results. The NEPSS database appeared to be a more complete record with a larger number of cases recorded for the period and more information on phage type and serovar. This demonstrated that this information was available for these isolates but had not been recorded in NDD.

Timely access to serovar and phage type information by PHUs is important as it assists the early identification and investigation of clusters and outbreaks. A major contributor to the delay in the receipt of Salmonella serovar and phage type details at the public health units was attributed to information being delivered by hard copy through the postal system. Electronic transmission of data has the potential to greatly reduce these delays.

An effective and efficient surveillance system for foodborne disease is important given the substantial burden of foodborne disease and its rapidly changing epidemiology. ${ }^{5}$ The capacity for foodborne disease surveillance in NSW has improved recently. The appointment of an additional epidemiologist has expanded the capacity for foodborne disease surveillance in NSW. Most serovar and phage type data is now available electronically to PHUs and PHUs are acting in a coordinated way.

The problems identified with data completeness and timeliness identified by this study would be further improved by:

1. Introducing electronic transmission of data from laboratories directly to NDD. This would reduce the current duplication of data entry and speed data delivery. Alternatively, a single database shared by laboratories and PHUs with appropriate security and access rules could reduce transmission delays and data errors. 
2. Reducing the complexity of sample handling. Having fewer laboratories involved in serovar and phage type determination would reduce the complexity of specimen and information flows between laboratories.

3. Routine monitoring of notification timeliness. The addition of further fields to the NDD to record the date of receipt of serovar and phage type results at the PHU would assist the monitoring of timeliness and remove the need for manual audits.

The NDD is currently undergoing a major review of both its structure and function. The feasibility of these and other suggestions for changes to the NDD to improve the surveillance of all notifiable conditions in NSW are being considered.

\section{ACKNOWLEDGEMENT}

This work was funded by the OzFoodNet enhanced surveillance program of the Commonwealth Department of Health and Ageing. We wish to acknowledge the contribution of Dr Jan Fizzell for her role in the collection and analysis of timeliness data for the paper audit of notifications to the Hunter PHU.

\section{REFERENCES}

1. Yohannes K, RocheP, Blumer C et al. Australia's notifiable diseases status, 2002: Annual report of the National Notifiable Diseases Surveillance System. Commun Dis Intell 2004;28:6-68.

2. Communicable Diseases Surveillance and Control Unit. Notifiable Diseases Manual. Sydney: NSW Department of Health, 2000.

3. Centers for Disease Control and Prevention. Updated Guidelines for Evaluating Public Health Surveillance Systems. MMWR 2001; 50 (RR13); 1-35.

4. Microbiological Unit, Department of Microbiology and Immunology. National Enteric Pathogens Surveillance System Human Annual Report 2000. Melbourne: University of Melbourne, 2001

5. Foodborne Diseases Working Party, Communicable Diseases Network Australia and New Zealand. Technical Report Series Number 2. Foodborne disease: Toward reducing foodborne disease illness in Australia. Commun Dis Intell December 1997: 2. Available online at www.health.gov.au/pubhlth/strateg/communic/tech/ foodbrne.htm 